\title{
The role of B lymphocyte stimulator (BLyS) in systemic lupus erythematosus
}

\author{
Michael P. Cancro, ${ }^{1}$ David P. D'Cruz, ${ }^{2}$ and Munther A. Khamashta²
}

${ }^{1}$ Department of Pathology and Laboratory Medicine, University of Pennsylvania School of Medicine, Philadelphia, Pennsylvania, USA. 2 Lupus Research Unit, The Rayne Institute, St. Thomas' Hospital, King's College School of Medicine, London, United Kingdom.

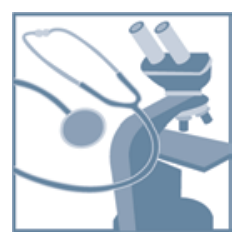

SLE, a chronic, multisystem autoimmune disorder with a broad range of symptoms, involves defective $B$ cell selection and elimination of self-reactive B cells. B lymphocyte stimulator (BLyS), a soluble ligand of the TNF cytokine family, is a prominent factor in B cell differentiation, homeostasis, and selection. BLyS levels affect survival signals and selective apoptosis of autoantibody-producing B cells. High levels of BLyS may relax B cell selection and contribute to autoantibody production, exacerbating the SLE disease state. This review discusses the mechanism of BLyS action on $B$ cells, its role in SLE, and specific targeting of BLyS in the treatment of SLE.

\section{The B lymphocyte stimulator family of cytokines and receptors}

B lymphocyte stimulator (BLyS, also known as the B cell-activating factor belonging to the TNF family, or BAFF; ref. 1) was identified as a novel TNF family ligand nearly 10 years ago (1-4) and has proven to be a key factor in the selection and survival of B cells. The BLyS protein is expressed by a wide variety of cell types, including monocytes, activated neutrophils, T cells, and DCs (5-7). BLyS is expressed as a cell surface protein that is then organized into a trimer, furin cleaved, and released into the circulation. Although standing levels of BLyS are constitutively generated, its expression and secretion can be potentiated by inflammatory cytokines, such as IL-2, TNF- $\alpha$, and IFN- $\gamma(6,8,9)$. A splice variant of BLyS that is inefficiently cleaved from the cell surface also exists $(10,11)$, and a 60 -mer form of soluble BLyS (BLyS-60) has been observed in mice $(12,13)$.

BLyS can bind to 3 receptors: BLyS receptor 3 (BR3; also known as BAFF-R), transmembrane activator-1 and calcium modulator and cyclophilin ligand-interactor (TACI), and B cell maturation antigen (BCMA). BLyS is the sole ligand for BR3, whereas TACI and BCMA each can bind either BLyS or another TNF family ligand known as a proliferation-inducing ligand (APRIL; Figure 1 and ref. 14). These ligand-receptor interactions vary in affinity: BLyS binds more strongly to BR3 than to TACI or BCMA, whereas APRIL displays the reverse affinity hierarchy. Recent in vitro evidence suggests that BLyS-60 binds to TACI with 100-fold higher affinity than the canonical, trimeric form (14). However, the existence of a soluble BLyS-60 remains to be determined in humans.

The 3 BLyS family receptors vary in their expression patterns and levels across different B cell subsets (Figure 2). During B cell

Conflict of interest: Michael P. Cancro has a sponsored research agreement with Human Genome Sciences Inc. David P. D'Cruz has received research grant support from Aspreva Pharmaceuticals Inc. and is participating in clinical trials in SLE led by Human Genome Sciences Inc., Hoffmann-La Roche Ltd., and Bristol-Myers Squibb Co. Belimumab is being developed by Human Genome Sciences Inc. and GlaxoSmithKline. Munther A. Khamashta has declared that no conflict of interest exists.

Nonstandard abbreviations used: AE, adverse event; APRIL, a proliferation-inducing ligand; BCMA, B cell maturation antigen; BCR, B cell receptor; BLyS, B lymphocyte stimulator; BR3, BLyS receptor 3; FO, follicular; MZ, marginal zone; SELENA, Safety of Estrogens in Lupus Erythematosus: National Assessment; SLEDAI, Systemic Lupus Erythematosus Disease Activity Index; TACI, transmembrane activator-1 and calcium modulator and cyclophilin ligand-interactor; TR, transitional.

Citation for this article: J. Clin. Invest. 119:1066-1073 (2009). doi:10.1172/JCI38010. development, the capacity to bind BLyS emerges concomitant with B cell receptor (BCR) expression (15), with higher binding found on $\mathrm{CD} 23^{+}$immature $\mathrm{B}$ cells emerging from the bone marrow (16). BLyS binding capacity increases through the transitional (TR) stages (17-19), and the highest expression of BR3 is found in the follicular (FO) and marginal zone (MZ) subsets of B cells (16). The activation of mature $\mathrm{B}$ cells leads to the up- or downregulation of TACI and BR3, with the exact effects dependent on the nature of the activating stimuli. Finally, long-lived plasma cells primarily express BCMA. The various receptor expression combinations and patterns foreshadow the biological actions of the different receptor-ligand pairs.

Among newly formed and mature primary B cells, BLyS-BR3 interactions are absolutely necessary for viability, because they provide essential and nonredundant survival signals $(14,20)$. The requisite for BLyS-mediated BR3 survival signals is first imposed during the TR stages of B cell differentiation, concomitant with the initiation of BR3 expression. This differentiative stage is the point at which newly formed B cells leave the bone marrow and enter the circulation and spleen. As detailed below, this stage is the last major checkpoint for elimination of potentially autoreactive primary B cells prior to their maturation $(21,22)$. BLyS signaling via BR3 antagonizes apoptosis at these stages, thus allowing further differentiation and entrance of cells into the mature, preimmune B cell populations - the FO and MZ compartments (20, 23). Moreover, BLyS signaling via BR3 continues to be required for $B$ cells to survive in these mature preimmune subsets.

In contrast to the actions of BR3, neither the development nor the maintenance of primary B cells requires TACI or BCMA (24, $25)$, as mice deficient in either of these BLyS family receptors generate normal preimmune B cell subsets (26). Instead, the data suggest that APRIL-TACI and APRIL-BCMA interactions influence antigen-experienced $B$ cell subsets, such as activated B cells, memory B cells, and long-lived plasma cells. For example, APRIL-TACI interactions can affect both survival and Ig class-switching characteristics of B cells during $\mathrm{T}$ cell-dependent immune responses, and TACI-knockout mice have subtle defects in T cell-independent responses and MZ B cell numbers (27). Roles for BCMA in antigen-experienced subsets are indicated by BCMA expression on long-lived plasma cells, as well as by evidence that the numbers of such cells are reduced in BCMA-knockout mice. In addition, 


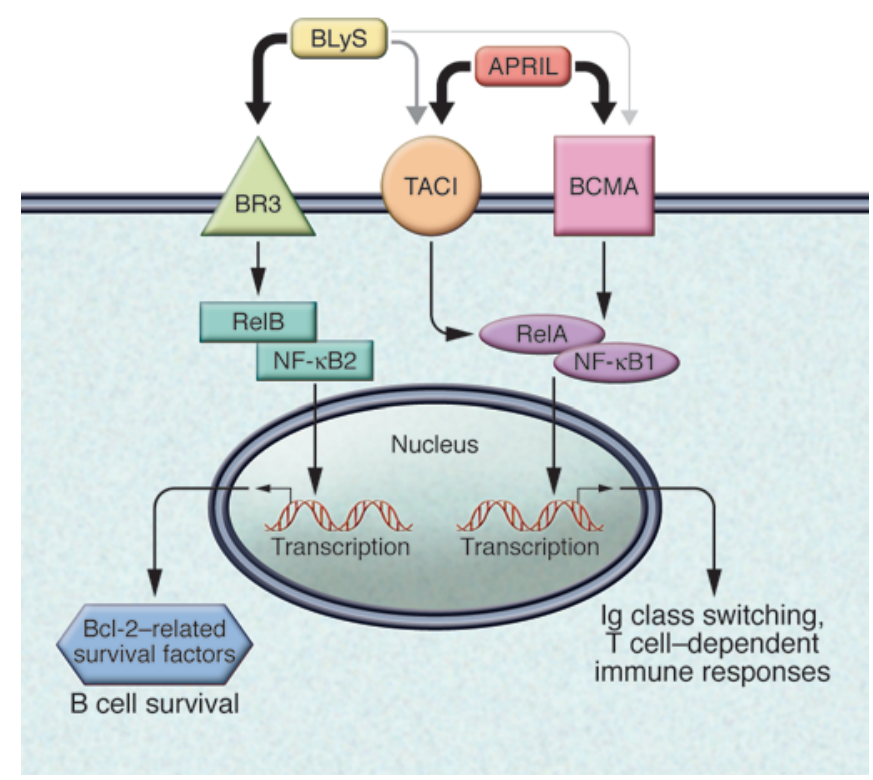

Figure 1

BLyS and APRIL ligands and receptors. BLyS is the only ligand for BR3, and both APRIL and BLyS bind to TACl and BCMA. APRIL binds to $\mathrm{TACl}$ and BCMA with higher affinity than does BLyS. Intracellular signaling from the ligand binding leads to $B$ cell survival and influences $B$ cell differentiation. These effects involve the activation of transcriptional regulatory factors such as RelA and RelB, which can regulate the expression of antiapoptotic proteins, including $\mathrm{Bcl}-2, \mathrm{~A}-1$, $\mathrm{Bcl}-\mathrm{X}_{\mathrm{L}}$, and $\mathrm{Mcl}-1$.

APRIL promotes plasma cell survival and is produced by some resident bone marrow cell types (28). These interactions may prove to be important considerations from the perspective of therapeutic intervention, because B cell subsets that rely on TACI and BCMA and their interaction with APRIL are likely to be relatively independent of BLyS and BR3.

\section{BLyS, B cell survival, and self tolerance}

The importance of BLyS-BR3 interactions in primary B cell maturation and survival was first noticed in BR3-deficient and BR3mutant mice (29-31), which display profoundly reduced peripheral $B$ cell numbers and drastically shortened life span of cells in the TR and mature $B$ cell subsets $(16,32,33)$. Moreover, these defects could be rescued by overexpression of the antiapoptotic Bcl-2 family member Bcl- $\mathrm{X}_{\mathrm{L}}$, suggesting that BLyS-BR3 interactions promote survival by preventing or overcoming apoptosis (34). Conversely, either exogenous administration or overexpression of BLyS rapidly led to B cell hyperplasia $(2,35)$ and autoimmune symptoms reminiscent of SLE and Sjögren syndrome (36). This relationship with autoimmunity has fostered intense investigation aimed at discovering how BLyS-mediated survival influences B cell tolerance.

Developing B cells that express autoreactive Ig receptors are purged at 2 checkpoints $(37,38)$. The first occurs in the bone marrow, where autoreactive cells either are eliminated through deletion or lose selfreactivity through a process called receptor editing (38-42). The second checkpoint is in the spleen and circulation, where TR B cells differentiate en route to becoming mature FO or MZ B cells (21, 22). In general, very strong BCR signals drive death or editing in the bone marrow, whereas less avid self-reactive clones become anergic and are then lost at the TR checkpoint $(15,43)$. Recent studies have shown that B cells expressing antibodies characteristic of humoral autoimmune syndromes, including anti-DNA and polyreactive specificities, are lost at the TR checkpoint (38).

The requirement for BLyS-mediated BR3 signals at the TR stages suggested that this might be the point at which BLyS affects tolerogenic processes. This has proven to be the case, because excess BLyS can cause autoreactive clones that normally die at this checkpoint to be rescued and allowed to mature (44-47). These findings indicate that anergic cells compete poorly for available BLyS and thus can survive only when BLyS levels are elevated. This ability to adjust tolerogenic stringency reflects cross-regulatory relationships between the intracellular signaling pathways used by the BCR and BR3 (48-51).

Together, these findings imply that BLyS serves 2 roles in the establishment and maintenance of primary B cells. First, it is the key regulator of primary $\mathrm{B}$ cell homeostasis, governing the overall numbers of mature, preimmune B cells by controlling their final step of differentiation and their life span. Second, it plays a central role in maintaining B cell tolerance by balancing the stringency of anergic cell elimination at the TR checkpoint against the need for additional preimmune B cells. Under normal conditions, this balance is achieved without substantial risk of autoreactive B cell maturation. However, in circumstances where B cell production from the bone marrow or the number of mature B cells falls outside these norms, or where BLyS levels are unusually high for extended periods, the likelihood that autoreactive cells will be allowed to mature is increased (43). Accordingly, the molecular mechanisms whereby BLyS-BR3 interactions mediate primary B cell homeostasis and the way these processes intertwine with mechanisms governing autoreactivity have been the subject of intense investigation over the last several years $(43,52,53)$.

The binding of BLyS to BR3 triggers intracellular signaling pathways that antagonize apoptosis in B cells, thus promoting survival. Mounting evidence indicates that members of the NF- $\kappa \mathrm{B}$ transcription factor family play central roles in these processes (54-56). Thus, BLyS signals via BR3 act primarily through activation of the so-called nonclassical NF-кB2 pathway, which facilitates upregulation of several antiapoptotic Bcl-2 family members.

The roles that BLyS family receptors and ligands play in activated and antigen-experienced subsets remain less understood. Based on the patterns of receptor expression among these cells, it is likely that TACI and BCMA will be the most prominent mediators of BLyS and APRIL activity in such cells. For example, TACI becomes elevated in B cells that have been activated through BCR or TLR stimulation, while BCMA is the prevalent receptor on long-lived plasma cells. Because APRIL binds these receptors with greater avidity than does BLyS, it is likely to exert greater influence than BLyS in these subsets. Most antigen-experienced B cell subsets appear to be independent of BLyS for survival $(28,57)$. Whether this indicates reliance on APRIL or instead reflects complete independence for BLyS family members remains unclear. In contrast to BR3, both TACI and BCMA signal through the classical NF-кB1 pathway, which has been generally associated with promotion of inflammatory responses and innate immunity $(43,55,58)$.

\section{Implications for B cells and BLyS in SLE}

SLE is a chronic multisystem autoimmune disease with a broad range of clinical manifestations $(59,60)$. SLE can present with many different symptoms, which include photosensitive skin 


\section{A B cell development and activation}

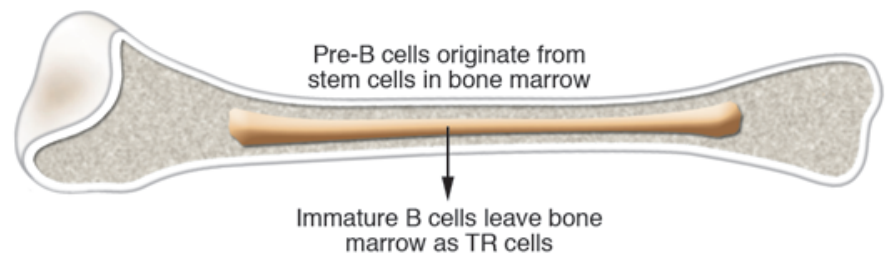

marrow as TR cells

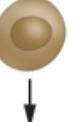

Mature, preimmune $\mathrm{B}$ cells recirculate through blood and lymphatics

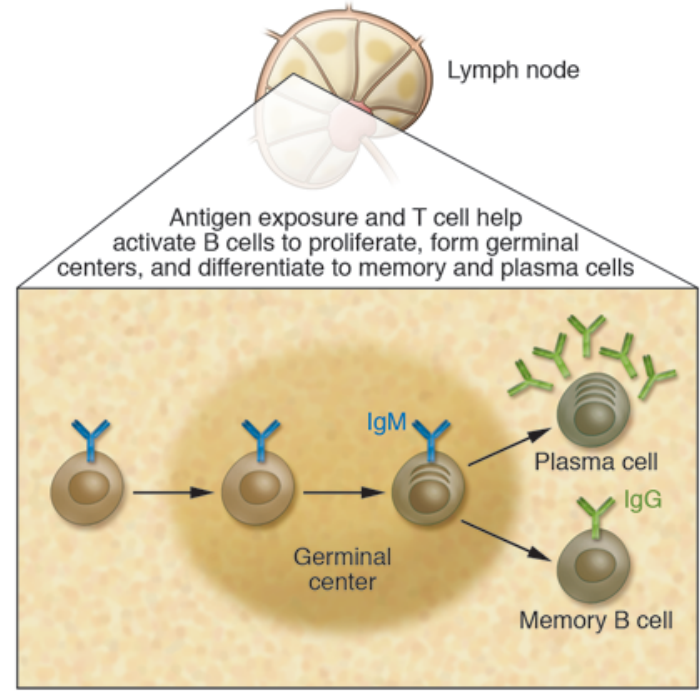

B B cell subsets and BLyS receptors

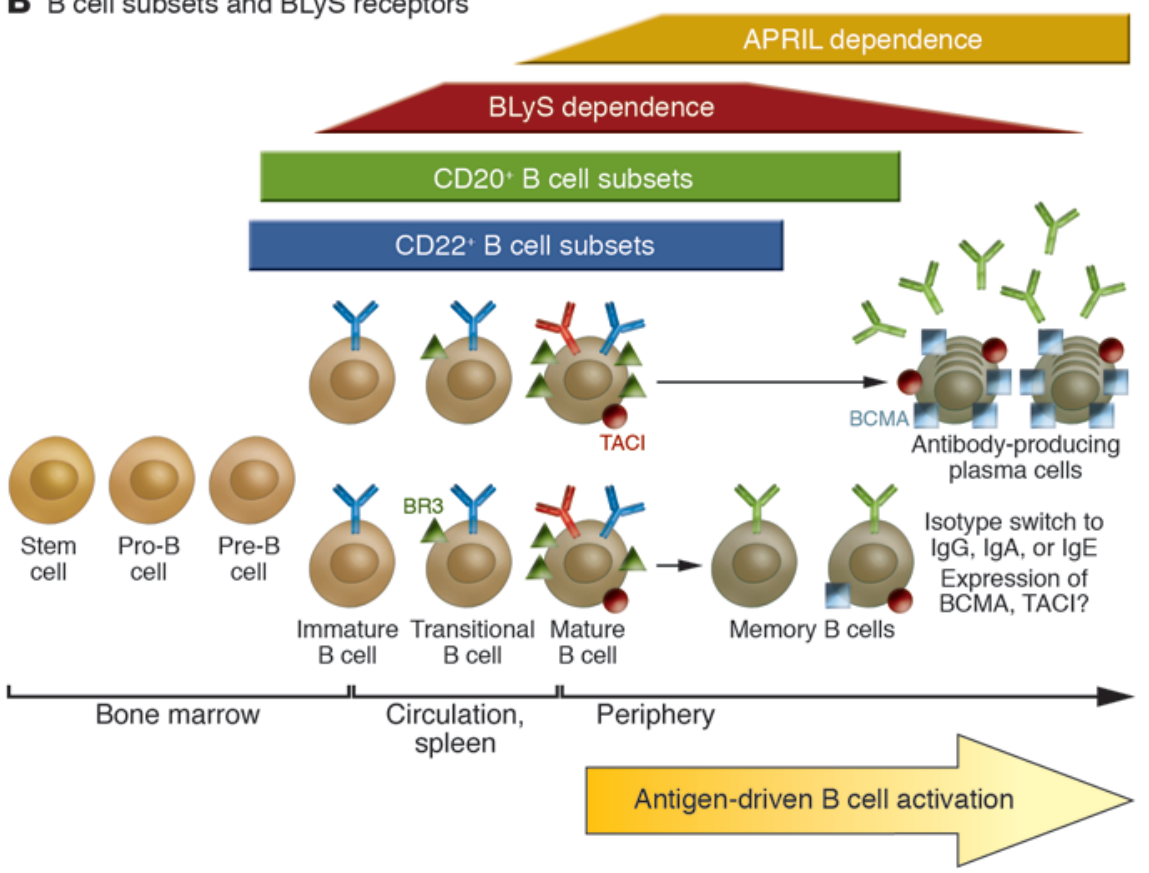

\section{Figure 2}

$\mathrm{B}$ cell differentiation and activation and BLyS receptor expression. (A) B cell development and activation. $B$ cells originate and complete initial phases of differentiation and maturation in the bone marrow. Immature B cells exit the bone marrow and move to the circulation as TR $B$ cells, then complete maturation in the spleen. If activated by antigen and T cell help, these mature preimmune $B$ cells will proliferate and form germinal centers, culminating in the generation of memory and plasma cells that make high-affinity, isotype-switched antibodies. (B) $B$ cell developmental stages and BLyS receptor expression. BLyS dependence ensues at the TR developmental stages, at which BR3 and $\mathrm{TACl}$ are first expressed. These receptors remain expressed among all preimmune populations. After antigen-driven activation, BLyS receptor expression shifts to predominantly TACl and/or BCMA expression among memory and long-lived plasma cells (the latter of which permanently reside in bone marrow).

rashes, discoid lesions, arthritis/arthralgia, nephritis, cardiac and pulmonary disease, and CNS disorders. Patients often present with nonspecific symptoms such as fatigue, headache, Raynaud phenomenon, oral ulcers, and mild hair loss (61-63), and diagnosis may be delayed. SLE occurs primarily in women, with African and Asian ancestry groups having the highest risk (59, 64-66). Although SLE is a chronic illness that most often results in debilitated health, it can be life threatening if major organs are affected (67). It is becoming increasingly clear that accelerated atherosclerosis associated with SLE may contribute to premature mortality $(68,69)$.

Serologically, SLE is characterized by the presence of high titers of autoantibodies and abnormal B cell activation and differentiation (70). Autoantibodies observed in SLE include those against a variety of nuclear antigens, including dsDNA, the ribonucleoprotein (RNP) complex Ro, the RNA-binding protein La, RNPs, the RNA molecule/protein complex Sm, the C1 complement component subunit $\mathrm{C} 1 \mathrm{q}$, and phospholipids. Complement levels are often reduced as a consequence of active disease, but in rare cases, they may be caused by a congenital complement deficiency (71). Autoantibodies have been detected 2-9 years before the onset of clinical features of SLE (72). However, the appearance of anti-dsDNA, anti-Sm, and anti-RNP antibodies suggest a peak in the intensity of the autoimmune response prior to the onset of clinical illness (73). 


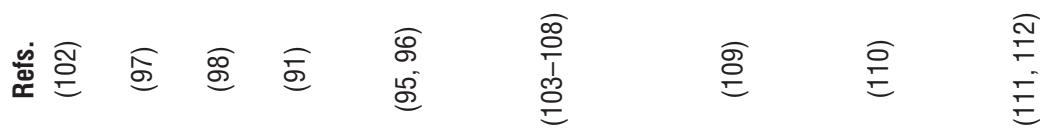
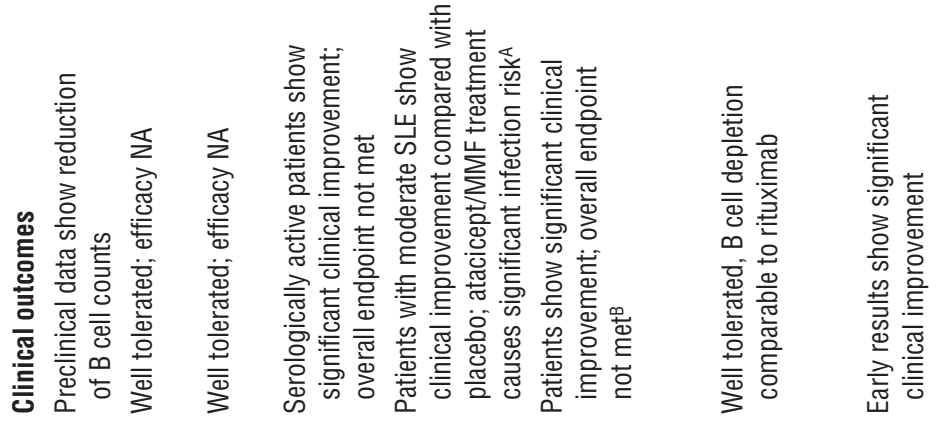

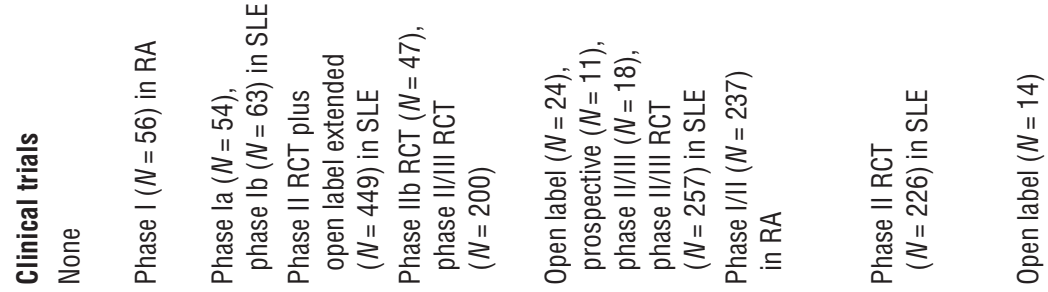

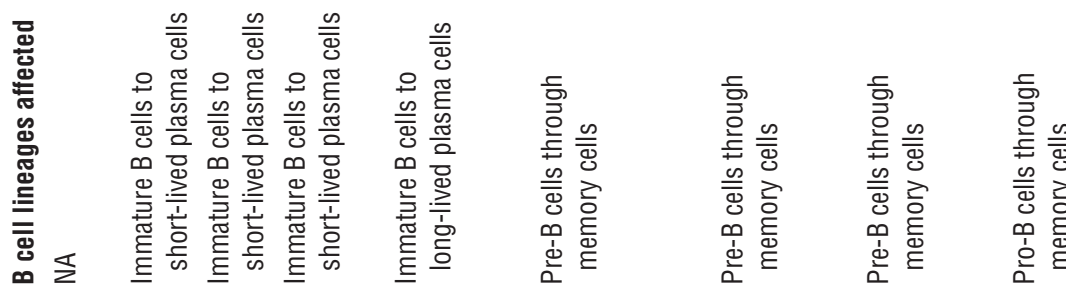

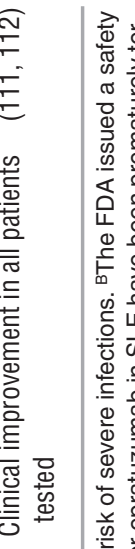

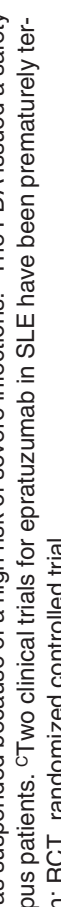

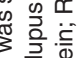

崩 흘

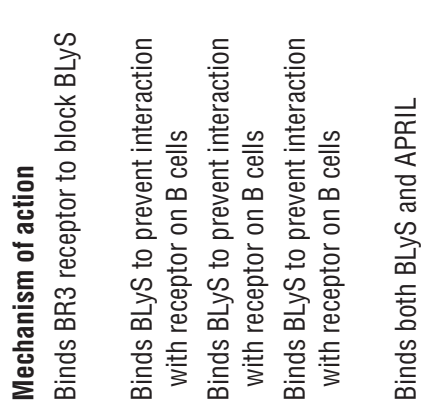

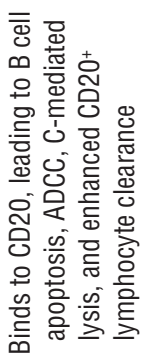

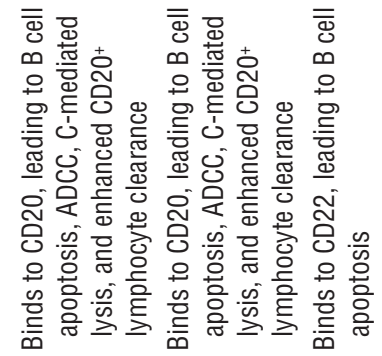

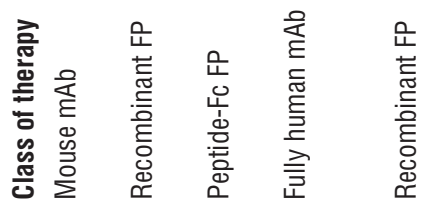

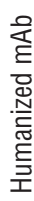

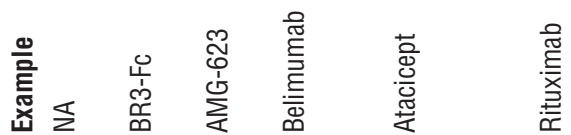

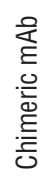

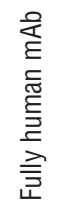

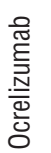

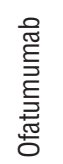

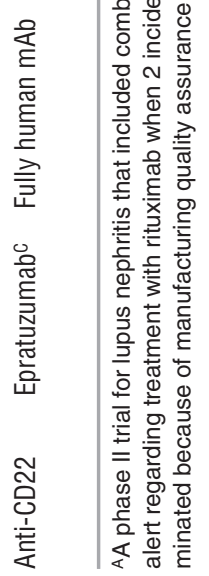

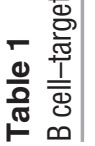

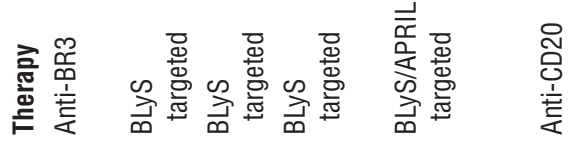


Numerous factors, both environmental and genetic, contribute to and trigger SLE development and autoantibody production. B cells and BLyS have recently emerged as important factors in the pathology of SLE, regardless of triggering factors. As noted above, BLySBR3 binding and signaling is critical for B cell survival or selection at the TR checkpoint. If this checkpoint is impaired or compromised in any way, it could allow moderately autoreactive or polyreactive $B$ cells to survive selection instead of undergoing elimination by apoptosis. A primary source of BLyS secretion is DCs, which are often located in mucosal, glandular, and cutaneous regions (74). Because much of the pathology of SLE is dermal and oral, it is reasonable to speculate that overactivity of the DCs may contribute to elevated serum levels of BLyS, and thus to SLE disease flares.

Elevated serum levels of soluble BLyS protein have been observed in patients with autoimmune disease, including those with SLE; evidence has shown that moderately autoreactive B cells in the spleen require higher concentrations of BLyS to survive the TR checkpoint than do B cells that produce non-self-reactive antibodies $(36,75)$. Additionally, mice treated with exogenous BLyS have shown increased numbers of antichromatin B cells, which suggests that high levels of BLyS contribute to elevated production of antiDNA antibodies, which are common in SLE patients (76). Transgenic mice that overexpress the BLyS protein develop autoimmune disorders similar to SLE and Sjögren syndrome (36). On the other hand, mouse models have confirmed that deletion of either BLyS or BR3 severely impairs B cell development beyond the TR developmental stages $(30,31,36,77)$. These data indicate that elevated BLyS levels may correlate with SLE disease activity. In addition, the development of class-switched autoantibodies in BLyS-transgenic mice appears to be $\mathrm{T}$ cell independent (78). This finding is interesting in light of the production of autoreactive B cells in human autoimmune disease; it suggests that there may be subsets of SLE or RA patients who develop autoantibodies in a $\mathrm{T}$ cell-independent manner and may benefit from BLyS-targeted therapy.

SLE has also been associated with defective clearance of apoptotic bodies. Apoptosis is a continual process that results in the release of subcellular debris and intracellular antigens, such as DNA, into the immune system. The clearing of apoptotic bodies is critical to preventing 2 events: first, uptake of the bodies by macrophages, which would present antigens to $\mathrm{T}$ and $\mathrm{B}$ cells, thus leading to an immune reaction against intracellular components (79); and second, presentation of intracellular TLR ligands to innate immune cells. The most common intracellular self antigens are ssDNA, dsDNA, dsRNA, and RNPs, among others, and each of these activates TLRs, resulting in elevated expression of BLyS (80). It is reasonable to speculate that increased levels of BLyS resulting from the presentation of self antigens to innate immune cells - which express BLyS upon antigenic stimulation - may further amplify the autoantibody production by B cells and exacerbate the SLE disease state. Moreover, IFNs are often profoundly dysregulated in SLE, and both IFN- $\alpha$ and IFN- $\gamma$ have been shown to induce BLyS expression $(5,81,82)$. A number of gene expression profile analyses have identified an IFN-related signature in SLE patients; more recently, SLE disease activity, as measured by Systemic Lupus Erythematosus Disease Activity Index-2K (SLEDAI-2K), was found to correlate with a strong IFN signature in a group of SLE patients $(83,84)$. Based on these data, it is tempting to speculate a potential cooperative action of BLyS and IFNs in the etiology of SLE. These observations suggest that BLyS may figure prominently in the development of autoimmune disease, particularly SLE, and may be a valid target for SLE therapy.

\section{BLyS-targeted therapy for SLE}

Elevated BLyS levels have been implicated in abnormal B cell development, including (but not limited to) autoantibody production, lymphadenopathy development (75), and lymphomas (85). By specifically antagonizing the modulatory protein, BLyS-targeted agents provide an effective way to control B cell activity (Table 1). BLyS-specific targeted therapy specifically affects early-stage $\mathrm{B}$ cells in the periphery without affecting late-stage compartments, such as memory or bone marrow plasma cells, and without compromising the immune system (86).

Belimumab. Belimumab is a fully human monoclonal Ab (IgG1) that binds soluble BLyS (86) and inhibits its binding to TACI, BCMA, and BR3 (Table 1). The specificity and affinity of belimumab for BLyS suggests that it may decrease B cell survival that results from an abundance of BLyS. Preclinical data demonstrate a role for belimumab in inhibiting BLyS: the administration of belimumab was found to inhibit the effects of BLyS in mice expressing the exogenous human protein, including decreases in spleen weight and serum IgA levels (86). This effect may stem, at least in part, from an attenuation of class switching in autoreactive $B$ cells. Recent nonclinical data have shown that anti-BLyS treatment appears to leave secondary immunity intact. A study of anti-BLyS treatment in mice determined that memory B cells, long-lived plasma cells, and secondary immune responses transiently rose, but remained whole, during and after exposure (87, 88). IgG-bearing memory $\mathrm{B}$ cells and natural Ab-secreting $\mathrm{B}$ cells were insensitive to anti-BLyS treatment, which suggests that they are BLyS independent and may be manipulated separately (57). A common phenomenon associated with BLyS-targeted therapies is the early transient rise in memory B cell numbers, which return to normal levels within 2 weeks after treatment (89).

The safety and efficacy of belimumab in SLE patients has been studied in a phase I randomized controlled clinical trial (90). In this dose-ranging study, belimumab treatment resulted in significantly greater percent reductions in $\mathrm{CD} 20^{+} \mathrm{B}$ cells than did placebo, consistent with the mechanism of action of this $\mathrm{Ab}$. This reduction was observed in patients treated with 1 or 2 doses of belimumab versus placebo-treated patients. A similar percent decrease in serum Igs was observed in belimumab-treated patients compared with placebo-treated patients; however, this decrease did not reach statistical significance. Overall, there was no difference in disease activity - as assessed by Safety of Estrogens in Lupus Erythematosus: National Assessment-SLEDAI (SELENA-SLEDAI) score - between the 2 treatment groups, but this may be because of the short term of the trial. Belimumab was well tolerated in the phase I study, with the majority of adverse events (AEs) being mild to moderate in severity, and the incidence of AEs similar in the placebo and belimumab treatment groups. In addition, there was no increase observed in the number of infections in belimumabtreated patients versus patients given placebo $(37 \%$ and $62 \%$ of the study population, respectively).

Belimumab appears to have a latency period before SLE disease improvement is seen, and clinical response was most evident in a subgroup of serologically active patients (91); each of these observations may account for the phase II trial not meeting its primary endpoint (Table 1). The long onset of efficacy for belimumab may be due to a slow off-time for BLyS at BR3, coupled with highly occupied receptors in SLE patients. A study of SLE patients showed reduced levels of available free BR3 on B cells in comparison with healthy controls (92). The reduced amount of 
available BR3 resulted in continuous receptor-ligand engagement. The slow off-time for BLyS at BR3, coupled with the abundance of BLyS, correlated with an increase in disease activity (92). BLyS also has a slow turnover rate, which may explain why there is a lag time or slower rate of disease response with belimumab treatment. The safety and efficacy of belimumab in SLE is currently being further validated in 2 large, global, phase III randomized controlled trials (BLISS 52 and BLISS 76; http://www.clinicaltrials.gov; trial nos. NCT00424476 and NCT00410384, respectively).

Atacicept. Atacicept (TACI-Ig) is a soluble, recombinant fusion protein of the human $\operatorname{IgG}_{1} \mathrm{Fc}$ and the extracellular domain of the TACI receptor that binds BLyS and APRIL and inhibits their action on B cells (Table 1). Similar to BLyS blockade, atacicept administration in a murine model of SLE led to delayed onset in SLE disease progression and reductions in B cell populations, spleen size, and DC activation $(93,94)$. However, in parallel to the anti-BLyS data above, it appears that TACI blockade reduces IgM-producing, but not IgG-producing, plasma cells. Whether this same effect occurs in humans remains to be seen.

In a dose-escalating phase I clinical trial (95), patients with moderate SLE showed a trend toward clinical improvement, as assessed by SELENA-SLEDAI scores, after treatment with atacicept. The small patient number $(n=12)$ precludes a definitive conclusion regarding the effect of atacicept on disease activity, although dosedependent reductions in mature and total $\mathrm{B}$ cell levels and Igs were observed. The reductions in B cells were sustained for up to 43 days in the single-dose group and up to 64 days in the repeated-dose group. Atacicept was well tolerated in this trial, with no significant differences in AE frequency or type observed between placebo and treatment groups. Although the number of infection-related events was similar in placebo and atacicept groups in the phase I trial, a recent phase II trial of atacicept in lupus nephritis was suspended because of a high risk of severe infections (96). A new phase II/III trial of atacicept in generalized SLE is currently open and recruiting patients (http://www.clinicaltrials.gov; trial no. NCT00624338).

Additional BLyS-targeted therapies. New biologic therapies targeted against BLyS are presently in development. These include BR3-Fc, AMG-623, and anti-BR3 Ab (Table 1). Of these, only BR3-Fc and AMG-623 have begun clinical trials, and while both appear to be well tolerated by patients, their efficacy in treating SLE and improving disease activity remains unknown $(97,98)$.

Other B cell-targeted therapies in SLE. Several therapies with a mechanism of action that targets B cells directly, in contrast to targeting BLyS, have also been tested in SLE patients and other autoimmune patients. Rituximab, ofatumumab, and epratuzumab have all demonstrated biological effect in SLE, while ocrelizumab has shown efficacy in RA (Table 1). Each of these has been shown to deplete the total population of peripheral $B$ cells with less specificity than BLyS-targeted drugs. Furthermore, the depleted state of B cells may persist for up to 10 months after treatment is terminated (99).

It is reasonable to speculate that reducing the number of B cells with anti-CD20 Ab therapy may effectively elevate the concentration of free BLyS, leading to increased incidence of autoreactive B cells surviving selection (43). A recent study of non-SLE B cell disorders showed that treatment with rituximab resulted in a 5 -fold increase in serum BLyS levels $(P<0.001$; ref. 100). In addition, recent clinical data reflecting the measurement of BLyS levels and autoreactive Abs in SLE during rituximab therapy $(n=25)$ showed that patients with higher BLyS levels at baseline were more likely to harbor anti-Ro and anti-RNP/Sm Abs after treatment; patients in this subgroup were significantly more likely to experience an SLE disease flare within 1 year of cessation of therapy (101). In this study, patients with lower BLyS levels prior to antiCD20 therapy fared better in the long term than did their higherBLyS counterparts (101). These data suggest that certain patients, especially those with high baseline BLyS serum levels, may not be the ideal candidates for rituximab therapy.

\section{Conclusion}

The definitive role of BLyS in SLE continues to be assessed. However, the elevated serum BLyS levels frequently observed in SLE patients, coupled with murine models of BLyS overexpression, suggest a significant role in the autoimmune process. The use of a fully human monoclonal Ab that binds soluble BLyS (i.e., belimumab) in serologically active SLE patients has resulted in reductions in disease activity and B cell populations without an observed increased risk of infection or major AEs. The continued clinical investigations into BLyS-targeted SLE therapy, as well as B cell deletion therapies, will aid in defining the role of BLyS and B cells in the development and perpetuation of SLE, as well as their place in future therapies for this heterogeneous patient population.

\section{Acknowledgments}

The authors acknowledge Patricia A. McChesney (Ogilvy Healthworld, New York, New York, USA) for editorial assistance.

Address correspondence to: Michael P. Cancro, Pathology and Laboratory Medicine, 284 John Morgan Building, University of Pennsylvania School of Medicine, 36th and Hamilton Walk, Philadelphia, Pennsylvania 19104-6082, USA. Phone: (215) 898-8067; Fax: (215) 573-2350; E-mail: cancro@mail.med.upenn.edu.
1. Schneider, P., et al. 1999. BAFF, a novel ligand of the tumor necrosis factor family, stimulates B cell growth. J. Exp. Med. 189:1747-1756.

2. Moore, P.A., et al. 1999. BLyS: member of the tumor necrosis factor family and B lymphocyte stimulator. Science. 285:260-263.

3. Shu, H.B., and Johnson, H. 2000. B cell maturation protein is a receptor for the tumor necrosis factor family member TALL-1. Proc. Natl. Acad. Sci. U. S. A. 97:9156-9161.

4. Mukhopadhyay, A., Ni, J., Zhai, Y., Yu, G.L., and Aggarwal, B.B. 1999. Identification and characterization of a novel cytokine, THANK, a TNF homologue that activates apoptosis, nuclear factor-kappa B, and c-Jun NH2-terminal kinase. J. Biol. Chem. 274:15978-15981.

5. Litinskiy, M.B., et al. 2002. DCs induce CD40-inde- pendent immunoglobulin class switching through BLyS and APRIL. Nat. Immunol. 3:822-829.

6. Nardelli, B., et al. 2001. Synthesis and release of B-lymphocyte stimulator from myeloid cells. Blood. 97:198-204.

7. Lavie, F., et al. 2008. Expression of BAFF (BLyS) in T cells infiltrating labial salivary glands from patients with Sjogren's syndrome. J. Pathol. 202:496-502.

8. Scapini, P., et al. 2003. G-CSF-stimulated neutrophils are a prominent source of functional BLyS. J. Exp. Med. 197:297-302.

9. Ogden, C.A., et al. 2005. Enhanced apoptotic cell clearance capacity and B cell survival factor production by IL-10-activated macrophages: implications for Burkitt's lymphoma. J. Immunol. 174:3015-3023.

10. Gavin, A.L., it-Azzouzene, D., Ware, C.F., and
Nemazee, D. 2003. \{Delta\}BAFF, an alternate splice isoform that regulates receptor binding and biopresentation of the B cell survival cytokine, BAFF. J. Biol. Chem. 278:38220-38228.

11. Gavin, A.L., et al. 2005. \{Delta\}BAFF, a splice isoform of BAFF, opposes full-length BAFF activity in vivo in transgenic mouse models. J. Immunol. 175:319-328.

12. Karpusas, M., et al. 2002. Crystal structure of extracellular human BAFF, a TNF family member that stimulates B lymphocytes. J. Mol. Biol. 315:1145-1154.

13. Liu, Y., Xu, L., Opalka, N., Kappler, J., Shu, H.B., and Zhang, G. 2002. Crystal structure of sTALL-1 reveals a virus-like assembly of TNF family ligands. Cell. 108:383-394.

14. Bossen, C., et al. 2008. TACI, unlike BAFF-R, is 
solely activated by oligomeric BAFF and APRIL to support survival of activated B cells and plasmablasts. Blood. 111:1004-1012.

15. Hardy, R.R., and Hayakawa, K. 2001. B cell development pathways. Annu. Rev. Immunol. 19:595-621.

16. Hsu, B.L., Harless, S.M., Lindsley, R.C., Hilbert, D.M., and Cancro, M.P. 2002. Cutting edge: BLyS enables survival of transitional and mature B cells through distinct mediators. J. Immunol. 168:5993-5996.

17. Allman, D.M., Ferguson, S.E., Lentz, V.M., and Cancro, M.P. 1993. Peripheral B cell maturation. II. Heat-stable antigen(hi) splenic B cells are an immature developmental intermediate in the production of long-lived marrow-derived B cells. J. Immunol. 151:4431-4444.

18. Allman, D., et al. 2001. Resolution of three nonproliferative immature splenic B cell subsets reveals multiple selection points during peripheral B cell maturation. J. Immunol. 167:6834-6840.

19. Loder, F., et al. 1999. B cell development in the spleen takes place in discrete steps and is determined by the quality of B cell receptor-derived signals. J. Exp. Med. 190:75-90.

20. Bossen, C., and Schneider, P. 2006. BAFF, APRIL and their receptors: structure, function and signaling. Semin. Immunol. 18:263-275.

21. Fulcher, D.A., and Basten, A. 1994. Whither the anergic B-cell? Autoimmunity. 19:135-140.

22. Fulcher, D.A., and Basten, A. 1994. Reduced life span of anergic self-reactive B cells in a doubletransgenic model. J. Exp. Med. 179:125-134.

23. Gorelik, L., et al. 2004. Cutting edge: BAFF regulates CD21/35 and CD23 expression independent of its B cell survival function. J. Immunol. 172:762-766.

24. Castigli, E., et al. 2004. Impaired IgA class switching in APRIL-deficient mice. Proc. Natl. Acad. Sci. U. S. A. 101:3903-3908.

25. Castigli, E., et al. 2005. TACI is mutant in common variable immunodeficiency and IgA deficiency. Nat. Genet. 37:829-834.

26. Varfolomeev, E., et al. 2004. APRIL-deficient mice have normal immune system development. Mol. Cell. Biol. 24:997-1006.

27. von Bulow, G.-U., van Deursen, J.M., and Bram, R.J. 2001. Regulation of the T-independent humoral response by TACI. Immunity. 14:573-582.

28. Belnoue, E., et al. 2008. APRIL is critical for plasmablast survival in the bone marrow and poorly expressed by early-life bone marrow stromal cells. Blood. 111:2755-2764.

29. Thompson, J.S., et al. 2001. BAFF-R, a newly identified TNF receptor that specifically interacts with BAFF. Science. 293:2108-2111.

30. Yan, M., et al. 2001. Identification of a novel receptor for B lymphocyte stimulator that is mutated in a mouse strain with severe B cell deficiency. Curr. Biol. 11:1547-1552.

31. Schiemann, B., et al. 2001. An essential role for BAFF in the normal development of B cells through a BCMA-independent pathway. Science. 293:2111-2114.

32. Harless, S.M., et al. 2001. Competition for BLySmediated signaling through $\mathrm{Bcmd} / \mathrm{BR} 3$ regulates peripheral B lymphocyte numbers. Curr. Biol. 11:1986-1989.

33. Lentz, V.M., Hayes, C.E., and Cancro, M.P. 1998 Bcmd decreases the life span of B-2 but not B-1 cells in A/WySnJ mice. J. Immunol. 160:3743-3747.

34. Amanna, I.J., Dingwall, J.P., and Hayes, C.E. 2003. Enforced bcl-xL gene expression restored splenic $\mathrm{B}$ lymphocyte development in BAFF-R mutant mice. J. Immunol. 170:4593-4600.

35. Gross, J.A., et al. 2000. TACI and BCMA are receptors for a TNF homologue implicated in B-cell autoimmune disease. Nature. 404:995-999.

36. Mackay, F., et al. 1999. Mice transgenic for BAFF develop lymphocytic disorders along with autoimmune manifestations. J. Exp. Med. 190:1697-1710.
37. Wardemann, H., et al. 2003. Predominant autoantibody production by early human B cell precursors. Science. 301:1374-1377.

38. Nossal, G.J.V., and Pike, B.L. 1975. Evidence for the clonal abortion theory of B-lymphocyte tolerance. J. Exp. Med. 141:904-917.

39. Nemazee, D., and Buerki, K. 1989. Clonal deletion of autoreactive B lymphocytes in bone marrow chimeras. Proc. Natl. Acad. Sci. U. S. A. 86:8039-8043.

40. Gay, D., Saunders, T., Camper, S., and Weigert, M. 1993. Receptor editing: an approach by autoreactive B cells to escape tolerance. J. Exp. Med. 177:999-1008.

41. Goodnow, C.C., et al. 1988. Altered immunoglobulin expression and functional silencing of selfreactive $B$ lymphocytes in transgenic mice. Nature. 334:676-682.

42. Radic, M.Z., et al. 1993. Residues that mediate DNA binding of autoimmune antibodies. J. Immu nol. 150:4966-4977.

43. Miller, J.P., Stadanlick, J.E., and Cancro, M. 2006. Space, selection, and surveillance: setting boundaries with BLyS. J. Immunol. 176:6405-6410.

44. Craxton, A., Draves, K.E., Gruppi, A., and Clark, E.A. 2005. BAFF regulates B cell survival by downregulating the $\mathrm{BH} 3$-only family member Bim via the ERK pathway. J. Exp. Med. 202:1363-1374.

45. Do, R.K., et al. 2000. Attenuation of apoptosis underlies B lymphocyte stimulator enhancement of humoral immune response. J. Exp. Med. 192:953-964.

46. Cyster, J.G., Hartley, S.B., and Goodnow, C.C. 1994. Competition for follicular niches excludes self-reactive cells from the recirculating B-cell repertoire. Nature. 371:389-395.

47. Fields, M.L., et al. 2003. The regulation and activation potential of autoreactive B cells. Immunol. Res. 27:219-234.

48. Davis, R.E., Brown, K.D., Siebenlist, U., and Staudt, L.M. 2001. Constitutive nuclear factor $\kappa \mathrm{B}$ activity is required for survival of activated B cell-like diffuse large B cell lymphoma cells. J. Exp. Med. 194:1861-1874.

49. Grech, A.P., et al. 2004. TRAF2 differentially regulates the canonical and noncanonical pathways of NF- $\mathrm{KB}$ activation in mature B cells. Immunity. 21:629-642.

50. Sasaki, Y., et al. 2006. Canonical NF-кB activity, dispensable for B cell development, replaces BAFFreceptor signals and promotes $\mathrm{B}$ cell proliferation upon activation. Immunity. 24:729-739.

51. Stadanlick, J.E., et al. 2009. Tonic BCR signals supply NFKB substrate for BLyS-mediated B cell survival. Nat. Immunol. 9:1379-1387.

52. Thien, M., et al. 2004. Excess BAFF rescues selfreactive $B$ cells from peripheral deletion and allows them to enter forbidden follicular and marginal zone niches. Immunity. 20:785-798.

53. Tsuiji, M., et al. 2006. A checkpoint for autoreactivity in human IgM+ memory B cell development. J. Exp. Med. 203:393-400.

54. Bishop, G.A., Hostager, B.S., and Brown, K.D. 2002. Mechanisms of TNF receptor-associated factor (TRAF) regulation in B lymphocytes. J. Lenkoc. Biol. 72:19-23.

55. Bishop, G.A. 2008. The multifaceted roles of TRAFs in the regulation of B-cell function. Nat. Rev. Immunol. 4:775-786

56. Ni, C.Z., et al. 2004. Key molecular contacts promote recognition of the BAFF receptor by TNF receptor-associated factor 3 : implications for intracellular signaling regulation. J. Immunol. 173:7394-7400.

57. Scholz, J.L., et al. 2009. BLyS inhibition eliminates primary B cells but leaves natural and acquired humoral immunity intact. Proc. Natl. Acad. Sci.U.S.A. 105:15517-15522.

58. Yang, M., et al. 2005. B cell maturation antigen, the receptor for a proliferation-inducing ligand and B cell-activating factor of the TNF family, induces antigen presentation in B cells. J. Immunol. 175:2814-2824.

59. D'Cruz, D.P., Khamashta, M.A., and Hughes, G.R. 2007. Systemic lupus erythematosus. Lancet. 369:587-596.

60. Simard, J.F., and Costenbader, K.H. 2007. What can epidemiology tell us about systemic lupus erythematosus? Int. J. Clin. Pract. 61:1170-1180.

61. Griffiths, B., Mosca, M., and Gordon, C. 2005. Assessment of patients with systemic lupus erythematosus and the use of lupus disease activity indices. Best. Pract. Res. Clin. Rheumatol. 19:685-708.

62. Hochberg, M.C. 1997. Updating the American College of Rheumatology revised criteria for the classification of systemic lupus erythematosus. Arthritis Rheum. 40:1725.

63. Tan, S.M., et al. 2003. Local production of B lymphocyte stimulator protein and APRIL in arthritic joints of patients with inflammatory arthritis. Arthritis Rheum. 48:982-992.

64. Bae, S.C., Fraser, P., and Liang, M.H. 1998. The epidemiology of systemic lupus erythematosus in populations of African ancestry: a critical review of the "prevalence gradient hypothesis." Arthritis Rheum. 41:2091-2099.

65. Danchenko, N., Satia, J.A., and Anthony, M.S. 2006. Epidemiology of systemic lupus erythematosus: a comparison of worldwide disease burden. Lupus. 15:308-318.

66. Petri, M. 2007. Monitoring systemic lupus erythematosus in standard clinical care. Best. Pract. Res. Clin. Rheumatol. 21:687-697.

67. Mok, C.C. 2006. Therapeutic options for resistant lupus nephritis. Semin. Arthritis Rheum. 36:71-81.

68. Doherty, N.E., and Siegel, R.J. 1985. Cardiovascular manifestations of systemic lupus erythematosus. Am. Heart. J. 110:1257-1265.

69. Petri, M. 2000. Detection of coronary artery disease and the role of traditional risk factors in the Hopins Lupus Cohort. Lupus. 9:170-175.

70. Davidson, A., and Diamond, B. 2001. Autoimmune diseases. N. Engl. J. Med. 345:340-350.

71. Green, J.R., Montasser, M., and Woodrow, J.C. 1986. The association of HLA-linked genes with systemic lupus erythematosus. Ann. Hum. Genet. 50:93-96.

72. Arbuckle, M.R., et al. 2003. Development of autoantibodies before the clinical onset of systemic lupus erythematosus. N. Engl. J. Med. 349:1526-1533.

73. McClain, M.T., et al. 2004. The prevalence, onset, and clinical significance of antiphospholipid antibodies prior to diagnosis of systemic lupus erythematosus. Arthritis Rheum. 50:1226-1232.

74. Jego, G., Pascual, V., Palucka, A.K., and Banchereau, J. 2005. Dendritic cells control B cell growth and differentiation. Curr. Dir. Autoimmun. 8:124-139.

75. Khare, S.D., et al. 2000. Severe B cell hyperplasia and autoimmune disease in TALL-1 transgenic mice. Proc. Natl. Acad. Sci. U. S. A. $97: 3370-3375$.

76. Hondowicz, B.D., et al. 2007. The role of BLyS/ BLyS receptors in anti-chromatin B cell regulation. Int. Immunol. 19:465-475.

77. Thompson, J.S., et al. 2000. BAFF binds to the tumor necrosis factor receptor-like molecule B cell maturation antigen and is important for maintaining the peripheral B cell population. J. Exp. Med. 192:129-135.

78. Groom, J., and Mackay, F. 2008. B cells flying solo. Immunol. Cell Biol. 86:40-46.

79. Munoz, L.E., et al. 2005. SLE - a disease of clearance deficiency? Rheumatology (Oxford). 44:1101-1107.

80. Sibilia, J. 2004. Novel concepts and treatments for autoimmune disease: ten focal points. Joint Bone Spine. 71:511-517.

81. Crow, M.K., and Kirou, K.A. 2004. Interferon-alpha in systemic lupus erythematosus. Curr. Opin. Rheumatol. 16:541-547. 
82. Harigai, M., et al. 2008. Excessive production of IFN- $\gamma$ in patients with systemic lupus erythematosus and its contribution to induction of B lymphocyte stimulator/B cell-activating factor/TNF ligand superfamily-13B. J. Immunol. 181:2211-2219.

83. Kirou, K.A., et al. 2005. Activation of the interferonalpha pathway identifies a subgroup of systemic lupus erythematosus patients with distinct serologic features and active disease. Arthritis Rheum. 52:1491-1503.

84. Nikpour, M., Dempsey, A.A., Urowitz, M.B., Gladman, D.D., and Barnes, D.A. 2008. Association of a gene expression profile from whole blood with disease activity in systemic lupus erythematosus. Ann. Rheum. Dis. 67:1069-1075.

85. Novak, A.J., et al. 2004. Expression of BLyS and its receptors in B-cell non-Hodgkin lymphoma: correlation with disease activity and patient outcome. Blood. 104:2247-2253.

86. Levine, M.H., et al. 2000. A B-cell receptor-specific selection step governs immature to mature B cells differentiation. Proc. Natl. Acad. Sci. U. S. A. 97:2743-2748.

87. Baker, K.P., et al. 2003. Generation and characterization of LymphoStat-B, a human monoclonal antibody that antagonizes the bioactivities of B lymphocyte stimulator. Arthritis Rheum. 48:3253-3265.

88. Benson, M.J., et al. 2008. Cutting edge: the dependence of plasma cells and independence of memory B cells on BAFF and APRIL. J. Immunol. 180:3655-3659.

89. Dorner, T., and Burmester, G.R. 2008. New approaches of B-cell-directed therapy: beyond rituximab. Curr. Opin. Rheumatol. 20:263-268.

90. Furie, R., et al. 2008. Biologic activity and safety of belimumab, a neutralizing anti-B-lymphocyte stimulator (BLyS) monoclonal antibody: a phase I trial in patients with systemic lupus erythematosus. Arthritis Res. Ther. 10:R109.

91. Chatham, W., et al. 2008. Belimumab (fully human monoclonal antibody to BLyS) improved or stabilized systemic lupus erythematosus (SLE) disease activity over 3 years of therapy [abstract]. Arthritis Rheum. 58:S573-S574.

92. Carter, R.H., et al. 2005. Expression and occupancy of BAFF-R on B cells in systemic lupus erythematosus. Arthritis Rheum. 52:3943-3954.

93. Ramanujam, M., et al. 2004. Mechanism of action of transmembrane activator and calcium modulator ligand interactor-Ig in murine systemic lupus erythematosus. J. Immunol. 173:3524-3534.

94. Ramanujam, M., et al. 2006. Similarities and differences between selective and nonselective BAFF blockade in murine SLE. J. Clin. Invest. 116:724-734.

95. Dall'Era, M., et al. 2007. Reduced B lymphocyte and immunoglobulin levels after atacicept treatment in patients with systemic lupus erythematosus: results of a multicenter, phase $\mathrm{Ib}$, double-blind, placebo-controlled, dose-escalating trial. Arthritis Rheum. 56:4142-4150.

96. Gonzalez, Á. 2008. Zymogenetics falls 29 percent after lupus trial is halted. October 28, 2008. http:// seattletimes.nwsource.com/cgi-bin/PrintStory. pl?document_id=2008319525\&zsection_id= $2003907475 \&$ slug $=$ zumogenetics $280 \&$ date $=$ 20081028.

97. Fleischmann, R., et al. 2006. BR3-Fc phase I study: safety, pharmacokinetics (PK) and pharmacodynamic (PD) effects of a novel BR3-Fc fusion protein in patients with rheumatoid arthritis [abstract]. Presented at the American College of Rheumatology Annual Meeting. November 10-15. Washington, DC, USA. Presentation 461.

98. Stohl, W., et al. 2008. Phase 1a single- and phase 1b multiple-dose studies of AMG 623 (an anti-BAFF peptibody) in systemic lupus erythematosus (SLE) [abstract]. Arthritis Rheum. 58:S565.

99. Dalakas, M.C. 2008. B cells as therapeutic targets in autoimmune neurological disorders. Nat. Clin. Pract. Neurol. 4:557-567.

100.Landau, D.A., Rosenzwajg, M., Saadoun, D., Klatzmann, D., and Cacoub, P. 2009. The BLyS/ BAFF receptor-ligand system in $\mathrm{HCV}$ induced B-cell clonal disorders. Ann. Rheum. Dis. 68:337-344.

101. Cambridge, G., et al. 2008. B cell depletion therapy in systemic lupus erythematosus: relationships among serum B lymphocyte stimulator levels, autoantibody profile and clinical response. Ann. Rheum. Dis. 67:1011-1016.

102. Lin, W.Y., et al. 2007. Anti-BR3 antibodies: a new class of B-cell immunotherapy combining cellular depletion and survival blockade. Blood. 110:3959-3967.

103.Leandro, M.J., Edwards, J.C., Cambridge, G., Ehrenstein, M.R., and Isenberg, D. 2002. An open study of B lymphocyte depletion in systemic lupus ery- thematosus. Arthritis Rheum. 46:2673-2677.

104.Leandro, M.J., Cambridge, G., Edwards, J.C., Ehrenstein, M.R., and Isenberg, D.A. 2005. B-cell depletion in the treatment of patients with systemic lupus erythematosus: a longitudinal analysis of 24 patients. Rheumatology (Oxford). 44:1542-1545.

105.Looney, R.J., et al. 2004. B cell depletion as a novel treatment for systemic lupus erythematosus: a phase I/II dose-escalation trial of rituximab. Arthritis Rheum. 50:2580-2589.

106. Merrill, J.T., et al. 2008. Efficacy and safety of rituximab in patients with moderately to severely active systemic lupus erythematosus (SLE): results from the randomized, double-blind phase II/III study EXPLORER [abstract]. Arthritis Rheum. 58:L12.

107. Merrill, J., et al. 2008. Design and baseline characteristics of patients in a randomized double-blind, placebo-controlled phase II/III study (EXPLORER) to evaluate the efficacy and safety of rituximab in patients with moderate to severely active systemic lupus erythematosus (SLE) [abstract]. Ann. Rheum. Dis. 67(Suppl. II):347.

108.Smith, K.G., Jones, R.B., Burns, S.M., and Jayne, D.R. 2006. Long-term comparison of rituximab treatment for refractory systemic lupus erythematosus and vasculitis: remission, relapse, and retreatment. Arthritis Rheum. 54:2970-2982.

109. Genovese, M.C., et al. 2008. Ocrelizumab, a humanized anti-CD20 monoclonal antibody, in the treatment of patients with rheumatoid arthritis: a phase I/II randomized, blinded, placebo-controlled, doseranging study. Arthritis Rheum. 58:2652-2661.

110. Ostergaard, M., et al. 2007. Ofatumumab (HumaxCD20), a fully human CD20 monoclonal antibody, in the treatment of rheumatoid arthritis: early results from an ongoing, double-blind, randomized, placebo controlled clinical trial [abstract]. Ann. Rheum. Dis. 66(Suppl. 2):124.

111. Dorner, T., et al. 2006. Initial clinical trial of epratuzumab (humanized anti-CD22 antibody) for immunotherapy of systemic lupus erythematosus. Arthritis Res. Ther. 8:R74-R85.

112.MSKReport.com. 2006. UCB, immunomedic phase III of epratuzumab in patients with systemic lupus erythematosus on clinical hold. Musculoskeletal report. September 27, 2006. http://www.mskreport. $\mathrm{com} /$ print.cfm?articleID $=961$. 